
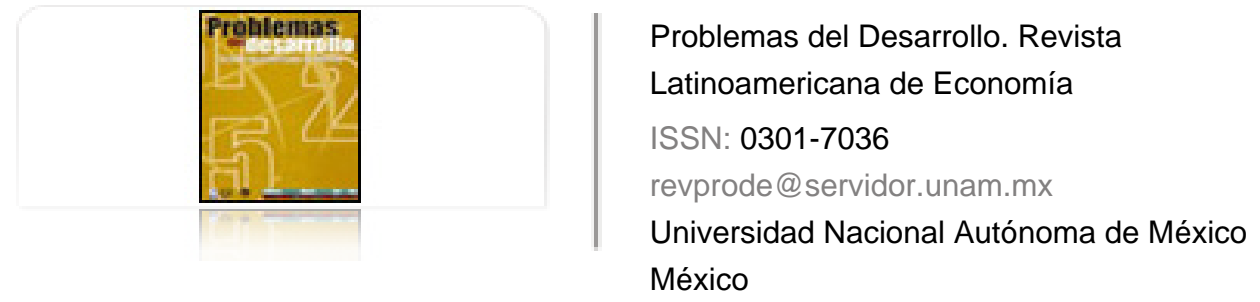

Rodríguez, Octavio

Claves para la agenda del desarrollo

Problemas del Desarrollo. Revista Latinoamericana de Economía, vol. 42, núm. 164, enero-marzo, 2011, pp. 5-23

Universidad Nacional Autónoma de México

Distrito Federal, México

Disponible en: http://www.redalyc.org/articulo.oa?id=11819774002

Cómo citar el artículo

- Número completo

- Más información del artículo

Página de la revista en redalyc.org

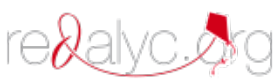

Sistema de Información Científica

Red de Revistas Científicas de América Latina, el Caribe, España y Portugal Proyecto académico sin fines de lucro, desarrollado bajo la iniciativa de acceso abierto 


\title{
Claves para la agenda del Desarrollo
}

\section{Octavio Rodríguez*}

Fecha de recepción: 23 de julio de 2010. Fecha de aceptación: 10 de enero de 2011.

\begin{abstract}
RESUMEN
El presente artículo parte de las concepciones de Celso Furtado respecto de la renovación de la agenda del desarrollo para América Latina. El autor analiza con especial interés y cuidado diversos conceptos, como los de desarrollo, identidad cultural, crecimiento autocentrado, inserción externa, cooperación internacional y asimetrías entre países. Insertos en una visión global de los sistemas culturales, esos conceptos constituyen elementos esenciales en una agenda del desarrollo, habida cuenta de las especificidades de los países subdesarrollados. Desde la óptica adoptada, se entiende que el subdesarrollo dificulta o inhibe la conformación de una identidad cultural propia, lo que a su vez tiende a entorpecer o impedir el desarrollo, tal como se lo entiende habitualmente en la literatura socioeconómica.
\end{abstract}

Palabras clave: Desarrollo, crecimiento económico, identidad cultural, crecimiento autocentrado.

\section{KEYS FOR THE AGENDA OF THE DEVELOPMENT}

\section{Summary}

This article is based on Celso Furtado's hypothesis of the renovation of the development agenda for Latin America. The author pays particular attention to analyzing the concepts of development, cultural identity, auto-centric growth, international insertion, cooperation and asymmetries. These concepts have come to be key ones in the development agenda. All of this is from the global viewpoint of cultural systems. In particular, this work is important because it aims to establish the basic concepts for sustaining the development agenda in under-developed countries. Seen in this way, under-development creates and impedes a proper cultural identity and therefore impedes development as defined in its traditional manner.

Key words: Development, economic growth, cultural identity, auto-centric growth

* Profesor emérito de la Facultad de Ciencias Económicas y de Administración de la Universidad de la República, Uruguay. 


\section{CLÉS POUR L'AGENDA DU DÉVELOPPEMENT}

\section{Résumé}

Cet article part de l'hypothèse de Celso Furtado sur le renouvellement de l'agenda de développement pour l'Amérique latine. L'auteur analyse avec un intérêt particulier les concepts de développement, d'identité culturelle, de croissance autocentrée, d'insertion internationale, de coopération et d'asymétries. Ces concepts deviennent les clefs de l'agenda du développement. Tout ceci depuis une vision globale sur les systèmes culturels. En particulier, ce travail est important parce qu'il essaye d'établir les concepts de base pour étayer durablement l'agenda du développement dans des pays sous-développés. Le sous-développement depuis cette optique, crée et interdit une identité culturelle propre qui, par conséquent, empêche le développement tel qu'il est défini dans sa forme traditionnelle.

Mots clés : développement, croissance économique, identité culturelle, croissance autocentrée

\section{CHAVES À AGENDA DE DESENVOLVIMENTO \\ Resumo}

O presente artigo parte da hipótese de Celso Furtado sobre a renovação da agenda de desnvolvimento para America Latina. $\mathrm{O}$ autor analisa com especial interesse os conceitos de desenvolvimento, identidade cultural, crescimento autocentrado, inserção internacional, cooperação e assimetrias. Estes conceitos são as chaves para a agenda de desenvolvimento. Tudo isso desde uma visão global sobre os sistemas culturais. Este trabalho é particularmente importante porque tenta estabelecer os conceitos básicos para sustentar a agenda do desenvolvimento em países subdesenvolvidos. O subdesenvolvimento desde essa ótica cria e impede uma identidade cultural própria o que impede, por tanto, o desenvolvimento tal qual este está definido na sua forma tradicional.

Palavras-chave: Desenvolvimento, crescimento econômico, identidade cultural, crescimento autocentrado.

\section{发展议程}

摘要

本文的假说是CelsoFurtado对拉丁美洲发展议程的重建设想。作者主要 探讨发展理

念，文化认同，自我为中心的增长，国际一体化，合作和不对称现象。 这些是当今发展议程中的关键词, 并要求以一个全球的视角来看待不同 的文化体系。

这项工作很重要, 因为它试图建立基本概念，以支持发展中国家的发展 议程。这一概念认为的不发达现象有助于创建文化认同感，同时也避免 陷入传统的文化理解模式从而阻碍自身发展。 关键词：发展, 经济增长, 文化认同, 自我为中心的增长 


\section{INTRODUCCIÓN}

El presente artículo tiene como fuente principal el Capítulo 15 de un libro de su autor titulado El estructuralismo latinoamericano. ${ }^{1}$ Dicho Capítulo se denomina "Hacia una renovación de la agenda del desarrollo". El mismo se configura como hilo conductor y como esquema de base de las ideas que pasaron a constar de dicho artículo, configurándose como las claves de una agenda temática sobre el desenvolvimiento, adecuada a países como los de América Latina.

\section{CONCEPTO DE DESARROLLO}

El concepto de desarrollo que Furtado adopta se refiere a cierta totalidad, que es el sistema global de la cultura. En otras palabras, Furtado teoriza sobre el desarrollo de sistemas culturales globales. Distingue en dichos sistemas elementos importantes a retomar.

\section{LA CULTURA MATERIAL Y EL ÁMBITO SOCIOPOLÍTICO}

La cultura material se refiere a los aspectos técnicos y económicos de esos sistemas. El ámbito sociopolítico, que constituye un primer aspecto de la cultura no material que incluye ideas y valores relativos a ese ámbito: el accionar de las clases y/o grupos que lo conforman. Por otro lado, el Estado juega a impulsar iniciativas concernientes a dicho ámbito. Estas iniciativas son expresiones de intereses de clases y grupos, derivan del uso de los márgenes de autonomía que el Estado habitualmente posee. Una estrategia conducente supone un papel relevante del Estado, y por ende, cierto grado de autonomía que permita ejercerlo. Otro aspecto de la cultura no material está constituido por las ideas y valores diversos de los sociopolíticos, que atañen a los más altos fines de la existencia

1 Cepal-Siglo xxi editores, México, 2006 (Ed. Brasil, Cepal-Civilizaçao Brasileira, 2009). El capítulo mencionado se titula "Hacia una renovación de la agenda del desarrollo". A dicho libro contribuyeron Óscar Burgueño, César Failache, Adela Hounie, Lucía Pittaluga y Andrea Vigorito, de la Facultad de Ciencias Económicas y de Administración de la Universidad de la República, y asimismo Gabriel Porcile, de la Universidad de Curitiba. 
humana. Son valores asociables a la reflexión filosófica, a la investigación científica, a la creación artística y a la meditación mística.

Las ideas de Furtado se refieren también al subdesarrollo, y más aún, tienen en la explicación del subdesarrollo su objetivo principal. Respecto a este tema, parece claro que si el desarrollo es el enriquecimiento de cualquier sistema cultural global, el subdesarrollo es el anverso, vale decir, la existencia de trabas que impiden ese enriquecimiento en ciertos sistemas culturales específicos. En otras palabras, se entiende que hay sistemas culturales que no logran el desenvolvimiento de una identidad cultural propia.

\section{IDENTIDAD CULTURAL}

El término no es más que una forma o una nomenclatura para hacer referencia a un sistema cultural específico, peculiar de un país o nación. Dicho término o categoría se introduce para decir que en países subdesarrollados como los de América Latina, el desenvolvimiento de una identidad cultural propia resulta obstaculizado. El subdesarrollo consiste en la existencia de trabas que impiden el enriquecimiento de una identidad cultural propia.

¿Por qué se da la traba recién aludida? Furtado la relaciona con la penetración de elementos de culturas foráneas; entiende que esa penetración compromete o perjudica la creatividad en los distintos ámbitos de la cultura. Aquí es importante considerar dos categorías.

\section{Creatividad e innovación}

El concepto se aclara si uno lo refiere al ámbito técnico. Se puede identificar una nueva tecnología como el conocimiento abstracto de las características de un nuevo bien o de un nuevo proceso productivo. Cuando el conocimiento en que el cambio técnico se incluye, se aplica en la realidad, se dice que él mismo dio lugar a una innovación.

Para Furtado, la creatividad y la innovación son fundamentales en los tres ámbitos de los sistemas culturales y postula que la penetración foránea inhibe ambas cosas en los tres.

Todo esto se hará más visible o más perceptible cuando se lo refiere a los elementos componentes de una estrategia de desarrollo para países como los latinoamericanos que han tenido notorias dificultades para impulsar su propia identidad. 


\section{CRECIMIENTO AUTOCENTRADO}

Se optó por la palabra crecimiento, en vez de hablar de desarrollo, por un entendimiento personal de que el concepto de desarrollo envuelve la consideración de relaciones sociales no económicas, mientras que los que se van a abordar en este tópico las deja de lado, atañen sólo a las de índole económica. La palabra autocentrado tiene en su base dos razones: la primera es que, para ser sostenido y eficiente, el crecimiento no puede dispensar un aumento continuado y significativo del mercado interno. La segunda es que tal crecimiento tampoco dispensa una presencia significativa y creciente de la propiedad nacional de los activos del país periférico de que se trate. Cubre lo tecno-económico, o sea, lo que Furtado denomina "cultura material".

Los temas cubiertos son: Ocupación, Inserción (estrategia mixta), Conducción macroeconómica, Cooperación y asimetrías, Consideraciones de conjunto.

\section{OCUPACIÓN}

En ella se diferencian empleo y subempleo. Para simplificar se deja de lado el desempleo abierto. El subempleo estructural y/o informalidad está constituido por la absorción de mano de obra a niveles de productividad en extremo reducidos. $\mathrm{Al}$ empleo se lo diferencia porque en él prevalecen niveles de productividad significativamente superiores. Hay un primer subgrupo, constituido por la ocupación en actividades donde la productividad se define como elevada. Sus niveles pueden ser los máximos factibles, los máximos permitidos por las tecnologías disponibles, o bien niveles próximos a éstos. Un segundo subgrupo comprende a la mano de obra ocupada en niveles de productividad intermedia que, aunque distantes de los de productividad elevada, son en extremo superiores a los del subempleo. En suma, para facilitar el análisis se diferencian tres capas técnicas: la de productividad elevada, la de productividad intermedia y el subempleo estructural.

En los días que corren y considerando un instante, lo que se percibe es que existe una mala asignación en tanto muchas actividades se llevan a cabo con productividad menor que la potencial. Esta cuestión de los niveles inadecuados de productividad tiene un aspecto dinámico de importancia clave. Las nuevas teorías del progreso técnico hacen prever una tendencia a la disparidad tecnológica. Para ir eliminando la mala asignación, es decir, para alcanzar una productividad cada vez mayor en más y más actividades, así como para contrarrestar la disparidad tecnológica se requiere diseñar e implementar políticas tecnológicas 
y productivas en diversas actividades, o en distintos conjuntos de actividades seleccionadas. Esto, como ya se dijo, hecho de forma de que las ocupaciones ampliadas se logren a productividad creciente. El éxito en esas políticas se verá facilitado si las mismas contemplan formar o mejorar un Sistema Nacional de Innovación (SNI) que ayude a diagnosticar las posibilidades existentes en materia de creación y/o incorporación de tecnología, de modo que facilite la diagramación de los caminos a seguir.

Vuélvase a la clasificación tripartita anterior, para brindar algo más de precisión a las políticas tecnoproductivas antes mencionadas.

Subempleo. En su reabsorción no habrá saltos. Se logrará ocupando mano de obra previamente subempleada en actividades de productividad intermedia. Una fórmula útil para la reabsorción es la que se ha llamado "transformación del atraso", en buena medida apoyada en la creación de microempresas, como se ha hecho visible en varios municipios del Estado de San Pablo (Brasil).

Productividad intermedia. La productividad deberá ir en aumento, no sólo por la reabsorción gradual del subempleo, sino porque la productividad podrá ir incrementándose con elevación de la productividad promedio, sin alcanzar los niveles de productividad elevada y/o máxima. En tanto en este grupo no se alcancen tales niveles, no habrá en ellos actividades competitivas, ni internamente ni de exportaciones. Para que puedan competir en el mercado interno -salvo casos como los muy altos costos de transporte- se requerirán ciertos niveles de protección, ya que se realicen por vía tarifaria, por subsidios o ventajas crediticias.

Productividad elevada. En este grupo, las actividades de productividad máxima factible serán por definición competitivas, tanto interna como externamente. Las de productividad elevada pero no máxima podrán también serlo, tanto en el caso ya mencionado de los altos costos de transporte, como por diferencias salariales. Pero también aquí habrá casos en los que la doble competitividad externa y/o interna requieran, ya sean medidas de promoción o medidas de protección. Por lo demás, el logro de la competitividad vía estímulos no excluye ni a los mismos bienes de productividad máxima. Esto porque las alzas de productividad son más aceleradas en los centros y habrá que cuidar de los niveles de productividad aun en los casos de productividad máxima, porque ese nivel puede dejar de serlo. Por eso mismo, habrá que incentivar el progreso técnico también en ellos, para que no surjan rezagos.

Viabilidad y eficiencia. El aumento de la productividad del trabajo continuamente impulsado en las actividades que componen los tres ámbitos antes dichos va de la mano con la incorporación de nuevas técnicas (ex-definitio, acompañado y suscitado por esa incorporación). Pero los aumentos de los empleos que se van alcanzando de ese modo son por sí mismos generadores de 
incrementos del mercado interno. Si bien se mira, estos últimos incrementos pueden percibirse como elementos integrantes de las condiciones de viabilidad de la estrategia propuesta: ella misma da lugar a aumentos del mercado necesarios para realizar la producción incrementada.

Por otro lado, los aumentos de la productividad del trabajo que definen dicha estrategia, brindándole su perfil esencial, pueden verse relacionados con el cumplimiento de condiciones de eficiencia; eficiencia en un sentido dinámico: implican que se irán logrando incrementos del excedente económico y con ello, aumentos del ahorro y de la acumulación de capital.

\section{INSERCIÓN (ESTRATEGIA MIXTA)}

En la publicación antes mencionada, el tema de la inserción internacional se trata, admitiendo que existe una tendencia al déficit comercial en las economías periféricas. Ahora se procederá a sintetizar los argumentos que constan de esa publicación, aceptando la misma hipótesis, esto es, aceptando que tal tendencia se verifica. Antes se habló de una estrategia que contemple el desarrollo de distintas actividades productivas y también las tecnologías que se adoptarán en cada una, pero orientándolas - producción y técnicas- hacia los problemas ocupacionales. Explícitamente, lo que se propuso es ir logrando la ocupación de la fuerza de trabajo a niveles crecientes de productividad. Ello implica dos cosas: lograr que en más y más actividades la producción se vaya acercando, y también vaya alcanzando el máximo técnicamente factible, con la eliminación gradual del subempleo estructural. Pero la estrategia propuesta tiene otra cara, que es la que se trata en este punto. Lo que se produzca y las técnicas con que se produzca, deberán también configurar una estrategia mixta (la expresión es de J. A. Ocampo). Es decir, una estrategia que contemple el estímulo a las exportaciones y, a la vez, el aumento sostenido de la producción para el mercado interno.

Las nuevas teorías del progreso técnico reconocen la existencia de una brecha tecnológica entre centros y periferia. A su vez, esta brecha tecnológica -por lo demás bastante visible- conlleva una tendencia al déficit comercial de la periferia. ¿¿Por qué? Porque su reiterado rezago tecnológico implicará la dificultad de expandir diversas actividades exportadoras de productos técnicamente avanzados para cuya producción la periferia carece de condiciones y tiene también fuertes necesidades de importaciones.

La disparidad tecnológica, y la tendencia al déficit comercial, constituyen la razón estructural de fondo, por la cual se niega la conveniencia de seguir un modelo de crecimiento extrovertido, es decir, el export-led growth que se propugna 
desde la perspectiva neoliberal, se postula la necesidad de un gran esfuerzo exportador, pero acompañado por un esfuerzo paralelo de aumento en la producción para el mercado interno.

Con algo más de detalle, la estrategia mixta puede ser concebida así:

- Existen conjuntos de actividades cuya producción se destina en gran medida al mercado externo, muchas de las cuales resultan competitivas en el punto de partida del diseño estratégico; otras habrán, también exportadoras, en las que se requiera adquirir condiciones de competitividad basadas en medidas temporarias de promoción.

- Existirán otras actividades cuya producción se destine al mercado interno; algunas virtualmente competitivas desde un comienzo, y deberán impulsarse otras actividades en las cuales se vayan ganando condiciones de competitividad con el tiempo, recurriendo para ello a medidas de protección, también temporarias. La promoción y la protección pueden darse de diversas formas, como recurrir a subsidios, tarifas, exenciones impositivas o a condiciones de crédito especialmente favorables.

Conviene enfatizar la palabra temporaria con la que se adjetivaron las propuestas de protección y promoción. Hace un tiempo, un diario publicó una foto de Prebisch, que al pie decía: "el hombre que cerró el continente, material y mentalmente". La realidad es que Prebisch propuso medidas de protección y de promoción temporarias y decrecientes por considerar que no habría progreso técnico continuado si no se imponía a la producción periférica el acicate de la competencia internacional.

Hoy, ese argumento adquiere especial valor porque el progreso técnico es muy rápido -vivimos una revolución tecnológica- lo que condiciona su absorción en la periferia. Para lograrla no se puede dispensar el acicate de la competencia. De ahí que en la propuesta de estrategia mixta se admita la necesidad de protección y promoción que favorezcan las exportaciones y la sustitución y que al mismo tiempo se insista en que ambas han de ser mínimas y decrecientes, o bien mínimas y transitorias. Igual, debe tenerse presente la necesidad de la apertura de los mercados centrales.

La estrategia mixta que conlleva expandir las exportaciones y "sustituir importaciones" puede ser vista o enfocada como una política tecnoproductiva orientada a obviar la tendencia al desequilibrio comercial. O mejor, bien entendida, ha de ser diseñada, y también rediseñada cada tanto, para lograr ese objetivo. Es por eso, por el desiderátum de lograrlo -evitar el desequilibrio y dar continuidad al crecimiento- que la estrategia mixta constituye una condición de viabilidad del crecimiento y/o desarrollo periférico. 
Por otra parte, en tanto con las políticas que constituyan la estrategia mixta se vaya cerrando la brecha tecnológica, es decir, se vayan alcanzando condiciones de competitividad internacional en más y más actividades, ya sean en las exportadoras o en las orientadas principalmente al mercado interno, se estará por eso alcanzando y ampliando las condiciones de eficiencia en las estructuras productivas periféricas.

Se vio anteriormente que las políticas ocupacionales son propuestas -y en la práctica, diagramadas- de forma que en ellas se cumplan condiciones de viabilidad y eficiencia. El tópico anterior pone de manifiesto que las políticas relativas a una inserción externa caracterizable como "estrategia mixta" añade condiciones de viabilidad y eficiencia propias de esta última.

En otros términos, globalmente considerada, la estrategia propuesta consiste, en esencia, en llevar adelante políticas productivas sectoriales que contemplen objetivos ocupacionales y también objetivos de compatibilidad intersectorial de la producción, con énfasis especial en la que debe darse entre exportaciones e importaciones. Desde ambas ópticas y en conjunto, ha sido delineada de modo que en ella vayan cumpliendo condiciones de viabilidad y eficiencia.

\section{CONDUCCIÓN MACROECONÓMICA}

Es necesario poner de manifiesto que el éxito de las estrategias de desarrollo tipificadas en los puntos anteriores no dispensan -sino que suponen- una conducción adecuada de las políticas macroeconómicas habitualmente consideradas de corto plazo: las políticas monetarias, cambiarias y fiscales.

Esa adecuación, en última instancia, no significa otra cosa que mantener equilibrios y evitar desequilibrios en esos ámbitos, de modo que el crecimiento de largo plazo - la transformación sostenida de márgenes de ahorro elevados en inversión real- no se vean comprometidos.

En verdad, en escritos relativamente recientes ligados a la CEPAL, de R. Ffrench-Davis y J. A. Ocampo, se demostró que un requisito indispensable de los equilibrios macro es el crecimiento sostenido de la economía. Es decir, demostraron que tales equilibrios no se obtienen por la sola facilitación (no ingerencia) de la operatoria de los mercados correspondientes, sino que, para sostener tales equilibrios en países como los de América Latina, es necesario mantener un crecimiento relativamente estable.

Pero más allá de esta alteración sustantiva de argumentos usuales, de trasfondo neoliberal, proponen otro en verdad de real importancia: el que los equilibrios macro no dispensan un sistema bancario y/o financiero adecuado en circunstancias que los de las economías de América Latina no lo son cabalmente. 
Una primera insuficiencia señalada por Ocampo es la estructura de esos mercados, en los cuales son escasos los ámbitos orientados a operaciones de largo plazo. Una segunda, son las limitaciones que en ellos presentan los mercados secundarios y/o de derivados.

Consideradas ambas cosas, se concluye que los mercados financieros periféricos poseen un tamaño reducido y eso los hace vulnerables a las presiones especulativas externas que vienen enfrentando. Puede admitirse que esta última debilidad, sintetizadora de las dos anteriores, no tiene otra salida u otra cura que llegar a la regulación de los mercados financieros internacionales o si se quiere, a los movimientos de capital financiero que se han puesto en práctica, y que han proliferado con la liberalización plena de aquellos mercados.

¿Por qué esto? Según se piensa, del estructuralismo se infiere la necesidad de regular dichos mercados en general y de admitir controles específicos muy cuidadosos para las economías subdesarrolladas. Ello, a raíz de que la tendencia al desequilibrio del lado real de la balanza de pagos - de la balanza comercial- no es compatible (no admite) cualquier grado de propiedad extranjera en dichas economías. Ésta tiene dos características: por un lado, es propiedad real de activos, es decir, IED. Por otro lado, es propiedad ideal: niveles de deuda externa que representan una parte "ideal" del valor del total de los activos periféricos. Los pagos consecuentes de ambos tipos de propiedades ahondarán las crisis latentes de la balanza en cuenta corriente que tienen en su base la tendencia al desequilibrio comercial.

No se sabe cabalmente qué decisiones serán tomadas. Hubo un claro rechazo a regular, cuando la crisis asiática, de ahí que avanzar en esa regulación requiera pensarla en términos geopolíticos. $V$.gr., una postura común de América Latina con apoyos adicionales en países subdesarrollados, incluso desarrollados. Ya se volverá sobre este tema cuando se aborde el de las crisis financieras y de la macro crisis actual.

\section{COOPERACIÓN Y ASIMETRÍAS}

\section{Cooperación}

a) Ya hemos planteado que en las condiciones periféricas, una estrategia de desarrollo conducente implica dos esfuerzos claves. Un esfuerzo de reabsorción del subempleo y de aumento continuado de la productividad del trabajo en múltiples actividades, a la vez que un esfuerzo de aumento de la producción en múltiples sectores.

Esos esfuerzos demandan en conjunto altos requerimientos de inversión, a raíz de que ambos envuelven sendos cambios estructurales -en la 
ocupación y en la producción- de amplitud y complejidad tales que se configuran como difíciles de lograr sin apoyo de la cooperación internacional. Apoyo que ha de presentarse con un doble aspecto: el ya seńalado en la inversión real, complementando las necesidades internas de un ahorro elevado y también la escasez de divisas emergentes, complementando su volumen en diversos periodos para viabilizar las importaciones requeridas por el crecimiento programado del PBI.

b) Éste es un argumento ya señalado por Prebisch en los documentos fundacionales. Planteaba pautar el recurso al ahorro externo a través del tiempo, por medio de relacionamientos financieros y comerciales ordenados -es decir, recurrir a déficits pero previendo sus pagos- que por lo mismo resultarían viabilizadores de un crecimiento intenso del PBI, con base en la expansión industrial. En otras palabras, viabilizadores de un cambio en el patrón de desarrollo que se basaría en dicha expansión.

c) Hoy existe una razón adicional que refuerza la necesidad de la cooperación en materia de inversión asociada a una revolución tecnológica en pleno curso. La misma comienza en los grandes centros pues es allí donde resulta más rápida.

Lo anterior implica que tienden a mantenerse o a surgir fuertes gaps tecnológicos que es necesario atenuar y también evitar en múltiples sectores. Para alcanzar estos objetivos se hace necesario recurrir al ahorro y a la inversión externa, tratando de asociarlos, a la adaptación y/o creación de tecnología. También puede considerarse que en la dirección de los mismos objetivos apunta el apoyo a los SNI, virtualmente relevante para los fines de adaptar y/o crear tecnología.

Además de estos gaps tecnológicos -respecto de los cuales ha de tenerse en cuenta que su conformación puede presentarse como reiterada- se encuentran presentes, y a veces se ensanchan, fuertes vallas de subempleo estructural. Hay que señalar que puede ser necesario sobrepasarlas o al menos atenuarlas para incrementar el mercado interno con cierta intensidad, o mejor, con una intensidad compatible con el aumento de dicho mercado, requerido por sectores que se tecnifican.

d) El apoyo financiero - el respaldo parcial de la inversión programada en el ahorro externo- es un primer aspecto de la cooperación. El segundo se refiere habitualmente al "trato especial y diferenciado".

De un lado, éste dice respecto al fomento a las exportaciones, por la vía de su promoción que puede tomar la forma de subsidios directos, exoneraciones fiscales o crédito privilegiado. También dice respecto al fomento a la sustitución de importaciones o mejor, a la producción para el mercado 
interno, por la vía de su protección, sea ésta tarifaria o ligada a otros medios, como los recién señalados.

El trato especial y diferenciado favorece el aprendizaje y la innovación, a través de la puesta en marcha de actividades que de otra manera no se podrían emprender. Pero se enfatiza que como ya se señalaba desde la época de Prebisch, lo que se propone son medidas de fomento temporarias, pues se considera imprescindible que en algún momento sobrevenga el acicate de la competencia (claramente tipificado, por ejemplo, en el caso de Corea).

e) Paralela y simultáneamente a la cooperación implicada en la promoción de las exportaciones periféricas o más precisamente, en la aceptación de las mismas por entidades internacionales y por los propios centros, puede pensarse que estos últimos adopten esa otra forma de cooperación, que sería la apertura de sus economías.

El efecto que puede esperarse es el mismo derivado de la promoción, pues se emprenderían actividades exportadoras virtualmente conducentes al aprendizaje y la innovación.

f) Puede suceder que la apertura de los centros traiga ventajas significativas, en tanto conduzca a enriquecer cadenas productivas, favoreciendo el desarrollo interno de determinadas ramas.

Un ejemplo: exportar ciertas partes de máquinas que exigen mercados de grandes dimensiones puede resultar clave para dar cauce a su fabricación interna e incentivar el desenvolvimiento de actividades conexas. Dicho ejemplo sugiere la posibilidad de que el aprendizaje e innovación involucrados en la apertura de los centros lleven a producir nuevas y mejores máquinas, en la periferia.

g) Hasta ahora se dijo que la promoción de las exportaciones periféricas y/o la mayor apertura de los centros, abren la posibilidad de producir y vender más bienes exportados, lo que va de la mano con más aprendizaje e innovación y, por ende, con el aumento o aceleración del progreso técnico periférico.

Hay, entonces, mayores o crecientes exportaciones de la periferia que permiten aumentar las importaciones requeridas para mantener un alto crecimiento interno. Pero estas importaciones son, como es claro, exportaciones de los centros, los cuales tenderán, por su lado, a más aprendizaje y más innovaciones, y por ende, a más posibilidades de progreso técnico.

b) Si bien se mira, la estrategia propuesta supone que la periferia regula la intensidad de sus relaciones internacionales, mientras que el centro ha de admitir una apertura en aumento y también a ritmo mayor.

Sin embargo, puede entenderse que de estos comportamientos asimétricos -benéficamente asimétricos- derivarían ventajas para ambos, más 
progreso técnico para los dos polos del sistema. Se trata de dos polos pertenecientes a un sistema único, de modo que cuando se da vitalidad al polo periférico también sobrevienen beneficios para el polo céntrico. En el conjunto del sistema habrá más progreso técnico y con él, más acumulación de capital y más crecimiento.

i) Prebisch generó la idea de una industrialización mancomunada. En la periferia, la industrialización implicaría importar menos de algunos bienes pero más de otros, con ventajas para los dos polos.

Ahora, la expresión puede transformarse en otra parecida: progreso técnico mancomunado. Se recrean condiciones para que éste aumente en la periferia, la misma exportará e importará más, y esas mayores importaciones redundarán en ritmos de aumento del progreso técnico en los centros que de otra manera no se darían.

Mirada desde otro ángulo, la expresión progreso técnico mancomunado alude a destrabar el desarrollo del polo periférico, vía continuados aumentos de la productividad que se asocian a la incorporación de progreso técnico, aprovechando la revolución tecnológica en curso. La expresión alude, asimismo, a que ello va de la mano con mayores exportaciones desde los centros hacia la periferia, lo que puede significar -se supone que significará- más progreso técnico y más desarrollo en dichos centros.

\section{Asimetrías}

a) Como se ha dicho, el "trato especial y diferenciado" de la periferia, de común acuerdo con medidas de apertura de los centros que apuntan a aprovechar las oportunidades que brinda el progreso técnico, favoreciendo el desarrollo de esos dos polos. Sin embargo, las políticas que se vienen implementando no apuntan en esa dirección.

En verdad, las políticas económicas internacionales se delínean con base en poderes económicos y políticos muy asimétricos, marcadamente orientados hacia los intereses de los grandes centros.

b) Un primer aspecto de esta asimetría se hace particularmente visible en la defensa de la agricultura de los centros, por la doble vía de la protección arancelaria y de los altos subsidios. Ésa ha sido una traba muy fuerte a las exportaciones periféricas en variados rubros del sector.

Otro aspecto del uso de ese mayor poder consistió en la puesta en marcha de un esquema de protección al acero, que llegó a tener vigencia, pero tan negativo y $\tan \sin$ razones que en poco tiempo se dio marcha atrás. 
c) Otro ámbito en que el poder asimétrico se pone de manifiesto atañe a la propiedad intelectual, en la cual se inscriben -entre otras cosas- los derechos de autor, las marcas de fábrica y de comercio, las patentes y la certificación vegetal. $^{2}$ Se reconoce las necesidades especiales de los miembros menos adelantados de aplicar leyes y reglamentos con la flexibilidad requerida para crear una base tecnológica sólida y viable. Es una cláusula muy genérica, de poca aplicación, pero que virtualmente admite apoyo público a los SNI. Hay una fuerte apropiación de técnicas o de los rendimientos que éstas producen. A veces se abren excepciones, no sin dura pugna, como en el caso del remedio contra la AIDS.

d) El mismo marco de mayor amplitud de la liberalización que acompaña los tiempos del Tratado de Marruecos (1994) en el que se estableció la OMC, tiende a acentuar los grados de libertad que alcanzan a la inversión extranjera directa. Aumentan las facilidades a que pueden acceder no sólo en materia de salida de dividendos, sino incluso en sendas facilidades jurídicas. Hay casos en los que las leyes y tribunales serán los de las casas matrices. Pero un punto esencial es que las ET's realizan esfuerzos de IxD y de CXT en esas casas y/o en los países de origen, en detrimento del progreso técnico en los países periféricos en donde obtienen lucros.

e) Un último aspecto -el más relevante- de las asimetrías de poder y de los problemas que trae consigo, atañe a la internacionalización y liberalización financieras, es decir, al trasiego descontrolado de recursos financieros entre países.

Tempranamente algunos teóricos supusieron que había sobrevenido la panacea, pues la plena libertad de mercado sería tan optimizadora como en general se supone en la economía para los mercados de bienes, y que el aumento de los recursos financieros invertibles favorecería el desarrollo en general, y en especial a los países más pobres y más carentes de capital (postura fuertemente negada por George Soros).

La liberalización e internacionalización financiera no trajeron los resultados optimistas esperados sino que dieron lugar a sucesivas crisis.

2 En 1967, en París se funda la ompi (Organización Mundial de la Propiedad Intelectual) que se empeña en favorecer o cuidar los intereses de los países en desarrollo. Por ejemplo, en su marco los países podían definir la duración de las patentes - no 20 años para todos los casos, como ahora-, establecer excepciones, y limitar patentes a las registradas internamente. Desde 1979, la diplomacia de USA se empeña en sustituir la OMPI por un organismo adscrito en el GATT. Lo logró en 1994 cuando éste se transforma en la omc. Se crea el Consejo de los Aspectos de los Derechos de Propiedad Intelectual relacionados con el Comercio (ADPIC). El nuevo marco definió obligación de patentes en inventos, pero adquiriendo valor universal. La inmensa mayoría se da en los centros. Pero hay una cláusula que de alguna manera busca generar resguardos para los países subdesarrollados. 
f) En años recientes, dos características destacan en dichas crisis.

La primera es su frecuencia, por lo demás reconocida por Nouriel Roubini (2008). Son 14 crisis en 10 años, pero hay también una alta frecuencia de crisis anteriores que se produjeron en países avanzados.

Empezando más temprano -en los años ochenta-principios de los noventa- se registran 8 crisis en 4 países avanzados: EEUU, Japón, Alemania y Escandinavia (contando a esta última como un solo país). Las mencionadas antes, en los países emergentes, fueron en más países y más tardías.

g) Falta mencionar la última: la crisis de hipotecas y créditos que comienza a gestarse hacia el 2000 y estalla en 2007-2008, llevando a la actual crisis financiera mundial.

El segundo elemento que debe considerarse es el vínculo de estas crisis y su frecuencia con la revolución tecnológica en curso, y con sus efectos en la instrumentación y manejo de los activos y sistemas financieros.

A veces se piensa en dicha revolución tecnológica como la causa de esas crisis, pero quienes han seguido de cerca el tema, sostienen que su causa clave se encuentra, en verdad, en el interés y el poder de los grandes bancos y entidades financieras, y en su incidencia en los gobiernos correspondientes. Fueron ellos -en realidad fue la operatoria liberalizadora a nivel internacional que pusieron en marcha- la que llevó a las crisis difundidas por el mundo.

Ese poder se concreta y se hace visible en la crisis asiática. Entonces, en el Grupo de los 7 se propuso reglamentar los movimientos internacionales de capital y/o el sistema que conforman, pero hubo oposición de Estados Unidos y Gran Bretaña que lo impidió; son, justamente, los países donde radican los principales y mayores bancos.

Pero aún aceptando el origen fundamental mencionado anteriormente, no desaparece la idea de que las nuevas técnicas, si bien no son la "causa última”, sí son -por decirlo de alguna manera- un mecanismo que permite que los componentes y operatoria de los sistemas financieros, al ampliarse y complejizarse, conduzcan a tendencias especulativas particularmente fuertes.

\section{CONSIDERACIONES DE CONJUNTO}

1. Las consideraciones anteriores permiten percatarse hasta qué punto -hasta qué grado extremo- la estrategia propuesta constituye un modelo abstracto. Un modelo en el cual las claves son la ocupación de la mano de obra a productividad creciente, y un patrón de inserción internacional que contemple, 
a la vez, la expansión de las exportaciones y el aumento de la producción para el mercado interno. Las metas recién referidas suponen la diagramación sectorial de la ocupación, y también la que dice sobre la producción, tanto de sectores exportadores como de los destinados al mercado interno.

Asimismo, se trata de una diagramación sectorial postulada bajo el supuesto de que los centros crecen con continuidad, facilitando el cambio de sus relaciones con una periferia que también crece. Se mostró también que, bajo esta doble condición, la estrategia cumple con condiciones de viabilidad y eficiencia.

¿Qué hacer -repensando el tema- cuando las condiciones de crecimiento dejan de cumplirse?

2. Lo que sucede es que hay que redefinir los sectores que conduzcan al cumplimiento de esas condiciones, contemplando los cambios ocurridos en lo real.

¿Cuáles serían los cambios previsibles? La generación de desempleo abierto -o mejor, su aumento- que es dejado de lado en la estrategia examinada.

Es claro que no se tratará sólo del desiderátum de ir eliminando el subempleo, sino que habrá que explicitar el trato del desempleo en la concretitud de la estrategia que se diseñará.

3. Un segundo cambio visible es el de la crisis financiera globalizada. Aun de la perspectiva periférica. Éste es otro problema clave para considerar que implica pugnar por la regulación de las relaciones financieras internacionales. No se sabe qué van a postular los grandes centros, o aun, qué van a querer imponer en esta materia. Sarkozy ha propuesto incluir en la discusión a los BRICS, pero parece claro que a los países periféricos cabrá plantear sus propias posturas que pueden apuntar a regulaciones particulares para su condición, como sería la posibilidad de acceder a formas específicas y favorables para el financiamiento en general o para el de exportaciones e importaciones.

Es claro que el éxito que se alcance en esta materia no puede pensarse en términos de las posturas y esfuerzos de países aislados. Se necesita una geopolítica, para cuya implementación los países de América Latina cuentan con condiciones históricas que la favorecen. Por otra parte, Brasil se va configurando como poseedor de condiciones positivas para su articulación dado que pertenece a los BRICs.

4. El tercer tema es la crisis hipotecaria (Estados Unidos) que ha traído consigo una crisis financiera compleja y grave y una crisis productiva que se hace más y más visible.

En esta materia existen posiciones encontradas. En un extremo, hay quienes piensan que es probable que se produzca una depresión intensa y generalizada, como en los años treinta. En el otro extremo, se dice que sí habrá 
recesiones, pero que serán relativamente suaves y breves y limitadas a los grandes centros.

Plantear una argumentación sugiere aceptar cierta hipótesis: la de quienes piensan que esta menor intensidad y duración se dará -no sin diferencias- tanto en la Unión Europea como en los Estados Unidos; asimismo, sostienen que las economías emergentes -China, India, Sudeste Asiático y también América Latina- verán caer sus niveles de crecimiento, pero lograrán desacoplarse en cierta medida de las economías centrales o principales, obviando males peores.

Si esto fuera así, se puede pensar que América Latina posee un primer ámbito para salvaguardar su necesidad de implementar políticas sectoriales favorables al progreso técnico, profundizando y ampliando la integración regional.

Éste tal vez sea el ámbito más fácil para conseguir progresos. Se puede pensar que la fuerte necesidad de buscar soluciones a las dinámicas sectoriales de exportaciones e importaciones ayudaría a intentarlos en el ámbito de lo latinoamericano o próximo a él. Los otros ámbitos de búsqueda de soluciones pueden estar en la profundización de las relaciones del Mercosur, o mejor aún, de América Latina con la Unión Europea, que se ha ampliado a nuevas subregiones promisorias en cuanto al desarrollo futuro.

El tercer ámbito para tener en cuenta es el de las oportunidades que brindan los países ricos y emergentes de Asia. Considerar conexiones con estos nuevos partners no implica ignorar ciertos puntos claves: la continuidad de la producción para el mercado interno y la selección de exportaciones hacia ellos y de importaciones desde ellos que, en conjunto con la producción antedicha, tenga en cuenta la búsqueda de una composición sectorial que ayude al progreso técnico todo lo que se pueda. Es decir, no se trata sólo de conseguir exportar sino de tener metas que no sean sólo la disponibilidad de divisas trabadas por el "cierre" de los grandes centros y la baja de precios. Se trata de planear esa disponibilidad, contemplando, además, el objetivo crucial del avance técnico cuidado sectorialmente.

Es claro que un programa en esa dirección no es nada fácil, y lo es todavía menos ya que no se debe dejar de considerar el futuro, tener en cuenta que en algún momento la crisis de los grandes centros tenderá a atenuarse, lo que permitirá reconsiderar las relaciones con ellos para un esquema de producción sectorial y de intercambio con el progreso técnico que se haya conquistando, y que abra la puerta a una mejora o aumento o aceleración del mismo.

5. En resumen: el modelo abstracto, la estrategia propuesta, sigue teniendo los mismos objetivos planteados antes de la crisis, los relacionados con superar 
la condición periférica: reabsorber subempleo y desempleo; producir exportaciones y bienes para el mercado interno; diagramando producciones sectoriales que sean decidida y fuertemente favorables al progreso técnico. El esquema abstracto es el mismo, aunque ahora se tendrá que considerar la marcha temporal de las relaciones con los nuevos ricos; también visualizar en el tiempo la reanudación de relaciones enriquecedoras con los grandes centros en crisis.

6. Lo anterior es el clímax de un finalle "sin brío", porque no se dice dónde hay que tomar medidas, ni con quién, ni en qué momento; se dice que habrá que planear todo eso no sólo para que las cuentas cierren, sino priorizando la vitalidad del progreso técnico, que es lo que verdaderamente se asocia a la salida de la "condición periférica" de los países de América Latina en el largo plazo.

7. Permite levantar una hipótesis optimista al reconocer la relativa precariedad del optimismo, en estos momentos. Toda crisis viene acompañada por oportunidades, y esta dialéctica de opuestos pudiera ser favorable, en el caso de América Latina, porque pueden vislumbrarse en esta área posibilidades especiales en el ámbito de la cultura no material, identificado como "sociopolítico" y también en aquel otro ámbito "más alto" de la misma, vinculado a posturas en los valores éticos.

\section{BIBLIOGRAFÍA}

Bielschowsky, R., "Celso Furtado e o pensamento econômico latino-americano", en: Luiz Carlos Bresser-Pereira e José Marcio Rego (orgs.). "A grande esperança em Celso Furtado: ensaios em homenagem aos seus 80 ańos", Sao Paulo, Editora 34, 2001.

Burgueño, O.; O. Rodríguez, "Desenvolvimento e cultura”, en Luiz Carlos Bresser-Pereira e José Marcio Rego (orgs.), "A grande esperança em Celso Furtado. Ensaios em homenagem aos seus 80 años", S. Paulo, Editora 34, 2001.

CEPAL, "América Latina y El Caribe: políticas para mejorar la inserción en la economía mundial”, Santiago de Chile: CepaL, 1995, LC/G.1800/Rev.1-P. , "Equidad, desarrollo y ciudadanía", Santiago de Chile, Cepal, 2000, LC/G.2071/Rev.1-P.

"Transformación productiva con equidad", Santiago de Chile: CEPAL, 1990, LC/G.1601-P.

Ffrench-Davis, R., "Macroeconomía para el crecimiento y globalización financiera: cuatro puntos estratégicos", Santiago de Chile, CePAL, 2004. 
Furtado, C., "El capitalismo global”, México, D.F., Fundación de Cultura Económica, 1999.

, "Creatividade e dependência na civilização industrial", Rio de Janeiro, Paz e Terra, 1978.

, "Cultura e desenvolvimento em época de crise", Rio de Janeiro, Paz e Terra, 1984.

,, "Dialéctica del desarrollo", México, D.F., Fondo de Cultura Económica, 1965.

, "Desenvolvimento e subdesenvolvimento", Rio de Janeiro, Fundo de Cultura, 1961.

, "Introdução ao desenvolvimento: enfoque histórico-estrutural", S.

Paulo, Paz e Terra, 2000.

, "O longo amanhecer", Rio de Janeiro: Paz e Terra, 1999.

, "O mito do desenvolvimento econômico", Rio de Janeiro: Paz e

Terra, 1974.

, "O subdesenvolvimento revisitado", en: Economía e Sociedade, (1)

1992.

Ocampo, J. A., "Raúl Prebisch y la agenda del desarrollo en los albores del siglo xxi", en: Revista de la CEPAL, núm. 75, diciembre, 2001.

, "Structural Dynamics and Economic Growth in Developing Countries". CEPAL, mimeo, 2003.

Rodríguez, O., "Heterogeneidad estructural y empleo", en: Revista de la CEPAL, núm. especial, oct., 1998.

, "Prebisch: actualidad de sus ideas básicas", Revista de la CEPAL, núm. 75, diciembre, 2001. 